\title{
Serious Gaming for Improvised Explosive Device Neutralization Training
}

\author{
Christopher C.K. Chan ${ }^{1}$, Alexander Ferworn ${ }^{1}$ and Const. Matthew Young ${ }^{2}$ \\ ${ }^{1}$ Dept. of Computer Science, Ryerson University, 350 Victoria St. Toronto, Ontario, Canada \\ ${ }^{2}$ Ontario Provincial Police, USAR/CBRNE Response Team, Bolton, Ontario, Canada
}

\begin{abstract}
An improvised explosive device (IED) is a "homemade" bomb intended to cause great harm when it explodes. The public safety task of identifying and neutralizing IEDs falls to military and police services often called explosive disposal units (EDU) who act to neutralize the threat associated with the IED either rendering it inoperable or destroying it safely. EDUs train in various aspects of explosive handling and investigation but are limited in the tools available for safely analyzing real world bombs. This paper describes a game based approach to IED training that employs an interactive 3D simulation to spatially identify key IED components of interest. We give an example of how this approach might be used and provide a preliminary evaluation of its potential effectiveness. We employ images formed from a Digital Imaging and Communications in Medicine (DICOM) system captured using Magnetic Resonance Imaging (MRI) technology to a virtual IED in a game. Empirical evaluation and EDU testimony suggest accurate representation of the IED and the potential efficacy of the proposed approach for successfully identifying components in the bomb for the purposes of EDU training.
\end{abstract}

\section{Introduction and Background}

An improvised explosive device (IED) is a "homemade" destructive device designed to destroy, incapacitate, harass, or distract [1]. Many commonly available materials such as fertilizer and common chemicals can be used as explosive materials in IEDs. They can be constructed in many forms, ranging in size and power from small and simple pipe bombs causing severe injuries to a few people to large and sophisticated devices capable of causing massive damage and loss of life.

In crisis and emergency management, IEDs have a long history, however, the Industrial Revolution created a surplus of preprocessed materials that enabled the development and diversity of IEDs [2]. Chemicals for explosives, and materials and products that can be modified to function as casings, shrapnel, triggering mechanisms and power sources have become readily available on a large scale. The potential number, variety and complexity of IED designs can make it difficult for EDU personnel to safely investigate a bomb's characteristics and effectively neutralize its threat without endangering themselves.

Regardless of design peculiarities, all IEDs are composed of a main explosive charge, a method of detonation, a triggering device, a power source and may include shrapnel [2]. There have been many examples of explosive charges constructed from everything from gun power [3] to fertilizer [4]. Detonators (the part of the bomb that sets off the main explosive charge) can be as small as a $1 / 2$ in. in diameter. Triggering mechanisms (the timer or communication mechanism for the bomb) can be readily improvised through the use of a clock or cell phone and depend on the intended use of the IED, available resources and the knowledge of the builder. The power source (supplying electricity to the other components) can be made from common batteries [5].

Our work focuses on visually determining regions of an IED that may resemble one of the key elements listed, for the purposes of bomb deactivation (neutralization). We propose an approach that involves the sensing of physical IEDs and rendering their virtual equivalents in software. For the purposes of our initial work, we consider IEDs hidden within a simple package (a cardboard banker box). Our intent is to provide a game mechanism for EDU members to practice, identifying key components of an IED and interacting with them to neutralize their threat in a safe environment. Figure 1 depicts our simulated IED that takes the form of a pipe bomb.

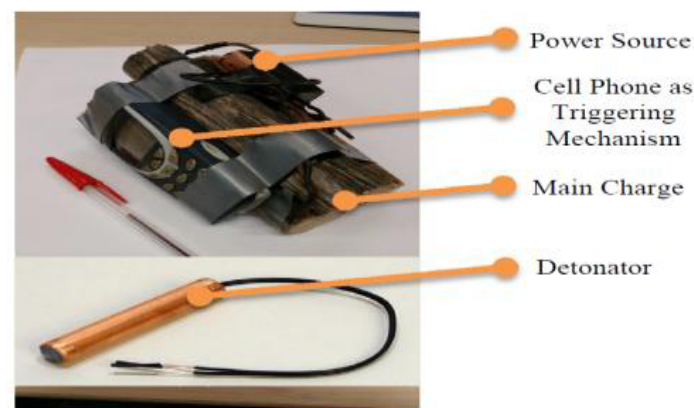

Figure 1. Simulated pipe bomb with a battery power source, cell phone trigger, commercial explosive main charge simulated by wooden sticks and a detonator. 


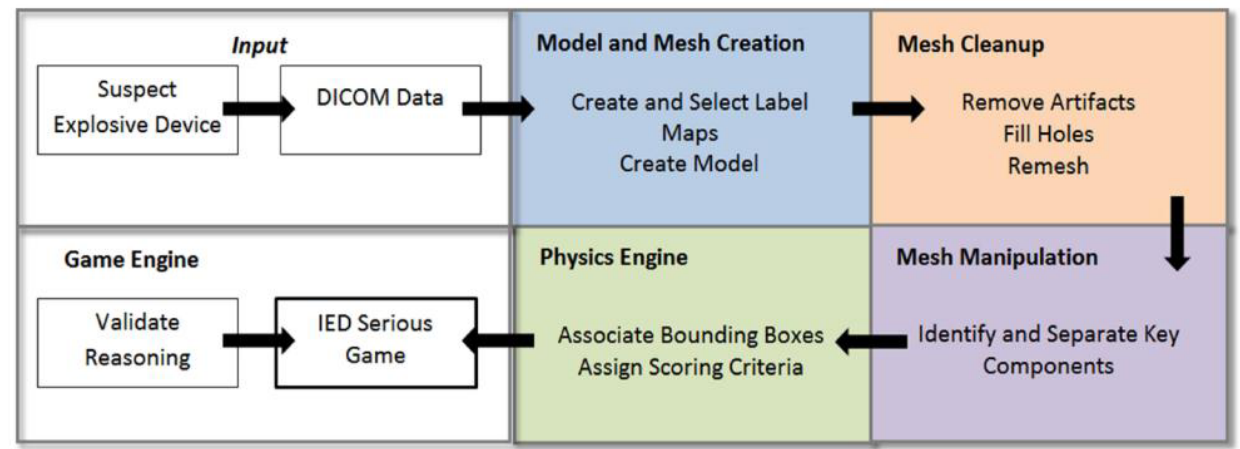

Figure 2. An overview of the proposed pipeline to create a virtual bomb from a real suspect explosive device real bomb.

Our goal is to use our virtual IED and concealing container as input into a serious game for purposes of EDU training, providing objective scoring concerning the neutralization task. In order to achieve our goal we must provide a physically accurate representation of the IED that maintains all spatial relationships between all of its components and the container it is in.

We utilize an interaction model browser [6] for exploring and visualizing relationships between structures that are often layered and visually occlude one another and we separate these models into independent parts for the 3D simulation within a game engine.

Figure 2 depicts the generalized overview of our pipeline. This work stems from our previous research in which we use data collected from an Unmanned Aerial Vehicle (UAV) fitted with an RGB-D sensor to create a high fidelity 3D point cloud model of scanned rubble terrain [7] and inject it into another serious game. Our previous work, in this broad area, focused on timesensitive operations with technology that can be applied to support USAR operations [7-9]. Stemming from this work, we present an approach to separate parts of an IED within a model and provide a game-based simulation that can potentially assist in the training of EDU personnel with the benefits of lowering exposure to actual IEDs, and providing visually and spatially accurate $3 \mathrm{D}$ model representations.

\section{EDU Operations and related work}

EDU operations involving a suspect explosive device (SED) normally begin by ensuring the three important safety factors are addressed; minimizing the amount of time spent in proximity to the device (since the device could be activated at any time by an integral timer, a remote trigger, or some other cause). Additionally, the response team and everyone else must comply with appropriate standoff distances calculated according to the apparent size of the IED. Finally, sufficient shielding must be provided to protect from a potential blast wave [10].

Essentially, the steps involved in dealing with a suspected SED-according to EDU professionals and the Federal Explosives Law and Regulations [11] involve many steps. One of these steps is to x-ray the SED (if possible) and determine where the power source/trigger is. It is important to make this determination to facilitate a means to neutralize the device by taking aim at one of the non-explosive components of the SED with a "disruptor" to disarm the SED. The process will be repeated if necessary.

The technology and software available to most EDUs are $\mathrm{x}$-ray scanners, $\mathrm{x}$-ray viewers [12], EOD disruptors [13] and various explosives to safely control and manage the SED through controlled detonation.

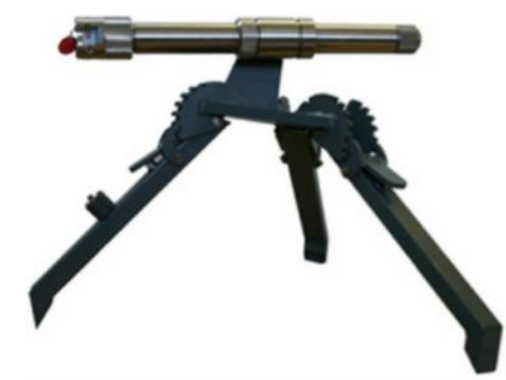

Figure 3. A 20mm Neutrex Waterjet Disruptor by Proparms [13].

Having taken an x-ray image, EDU personnel attempt to quickly determine if a SED is an IED, and if it is an IED, identify the primary components. They use this information to determine the best approach to neutralizing the device.

The closest related work to our proposed approach is represented by the Scanview [12] software package--an application developed by Scanna MSC in the UK. Scanview provides a number of image manipulation and enhancement tools that can help EDU specialists identify the components in an IED, using a 2D image. It provides a "pseudo-3D" feature that can provide some depth representation of the scanned image.

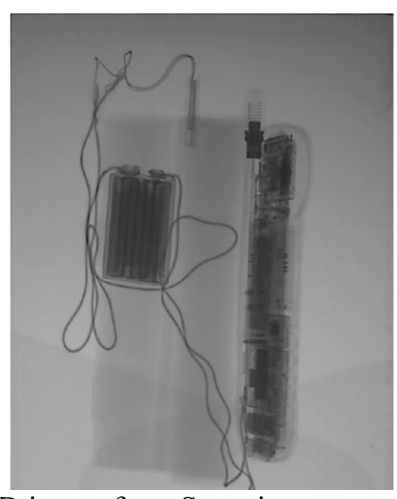

Figure 4. The 2D image from Scanview. 
Serious games have been developed in a number of arenas such as defense, education, scientific exploration, health care and emergency management [14]. The main purposes of a serious game can be to train, educate or promote learning for a particular topic. A serious game requires a player to solve a problem while being evaluated (rewarded) through a scoring mechanism. Common games range from air defence [15] to forms of military training [16] to rehabilitation [17]. Serious games provide realism and presence in a virtual environment sometimes employing physics [18-19] to facilitate reality simulation. A physics engine computes how physical objects should move and interact with each other within the game.

Previous work related to IEDs focuses on locating IED threats [20-21], determining the blast effects of IEDs [22], IED threat assessment [23], and determining where IEDs might be placed [24]. The closest related work to interactive $3 \mathrm{D}$ simulation is USARSim [25]. Few software systems have been created to aid in neutralizing an IED.

Our work revolves around the use of non-traditional, noncontact sensing and recording of physical IED devices, their modeling and interaction with trained operators who act as players in serious game settings in which a player is asked to neutralize the threat and is scored on their ability to do so using devices within the game.

\section{Methodology}

Our proposed algorithm is comprised of three steps. The first step involves scanning the IED in a process we call the model creation pipeline (MCP). In contrast to the high fidelity point cloud framework described in our previous work [7-8], this process utilizes open source projects, Slicer 3D [26], Blender [27] and Aperio [6] to convert Digital Imaging and Communications in Medicine (DICOM) data into separate mesh models as object files compatible with game engines such as Unity [28] or Unreal [29] for interactive 3D simulation. This MCP allows for the ability to inject arbitrary real models into a game engine and reason about them.

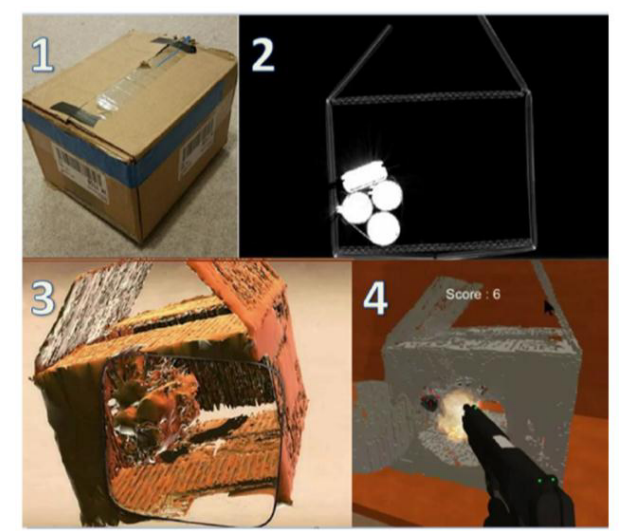

Figure 5. 1). A simulated IED within a banker box; 2). A DICOM cross sectional image of the bomb; 3). The bomb in Aperio for the purposes of revealing occlusions and separating the bomb into components; 4). The model inside the game engine for interactive training.
The second step is to create an injectable model of IED components in order to make a virtual representation of the entire IED within a game engine. In addition, other models of necessary tools and artifacts can be created to interact with the virtual IED. One such tool - known as a disruptor - is used to remove key components of an IED by accurately firing a bolt of water at them. In our simulation, we model the $20 \mathrm{~mm}$ Neutrex disruptor sold by Proparms [13]. In addition, we create a model of what we call "the rig" which is an assemblage of the objects around the IED. As all the models are physically accurate and the game engine supports physics-based interactions, we are able to reason about them inside the game. The results of reasoning take the form of data such as the distance from the Neutrex disruptor to the container and the angle to the target with respect to the normal plane of the disruptor. The reasoning occurs by the player interacting with the artifacts inside the game simulation and can be validated against a real-world IED and rig.

The third part of the system is a game scoring algorithm which measures the effectiveness of the disruptor's target location with a projectile blast in the game. We take advantage of known heuristics. For example, if the disruptor fails to hit the desired target the score will be low while hitting the target leads to a high score. Of course, hitting the main charge, causes the game to end abruptly.

The simulation provides feedback to a player through scoring points as to the effectiveness of their targeting. We suggest that this interactive part of the simulation can be used to safely train response personnel by providing visual cues and feedback for important and specific regions of interest of simulated IEDs.

Our initial system is informed by input from subject matter experts from the Ontario Provincial Police (OPP) UCRT from their Chemical, Biological, Radiological, Nuclear explosive (CBRNe) team and the Deltic Group [30] who supply a range of specialty equipment such as $\mathrm{x}$-ray technology for CBRNe applications.

Based on initial feedback, we feel that future versions of our training simulation may be able to augment the training for responders to identify bomb neutralization targets - supplementing the identification process that ordinarily is performed in the real world. The advantage in using our proposed methodology is that the simulated IEDs can be easily shared between EDUs throughout the world. Once scans are obtained and injected into a game, EDU reasoning about the virtual model as a precise physical representation of the real model is facilitated in a safe environment.

Our initial work is described below.

\subsection{Create Representative IED}

The real world inert IED was constructed using a 9V battery as a power source, an old cell phone as a trigger, small wooden pieces as the main charge (see figure 1) with the assembly wrapped in electrical tape. These components were chosen from commonly available material with varying densities which are characteristic of pipe bombs according to CBRNe team members [30]. On $\mathrm{X}$-ray technologies [12] (see figure 4) components are 
differentiated by their densities, the darker the component is, the denser the material. In our algorithm, the components are segmented based on signal intensity.

\subsection{Place the IED within a representative container}

The inert pipe bomb was placed within a small banker box with dimensions of $21 \mathrm{~cm} \times 25 \mathrm{~cm} \times 16 \mathrm{~cm}$. Packing air bags were placed around the pipe bomb as supports to avoid movement in of the IED in transit.

\subsection{Scan the IED within the container}

Magnetic Resonance Imaging (MRI) technology was used to capture data of the container and the bomb. MRI is one of many modern diagnostic imaging techniques that produces cross-sectional images of an entity using energy from an oscillating magnetic field at the appropriate resonance frequency [31]. This process excites hydrogen atoms and causes them to emit a radio frequency signal which is measured by a receiving coil. The MRI machine decodes densities of different components by the rate at which excited hydrogen atoms return to the equilibrium state by varying the main magnetic field using gradient coils.

This method of scanning has many advantages for EDU personnel over the current $x$-ray methodology as there is no risk of radiation exposure. In addition, actual scans take less than 30 seconds and an operator can change the imaging plane without moving the object being scanned. However, the technology cannot readily be used for EDU operations as no magnetic components can exist within the IED being scanned and MRI machines are exceedingly expensive, relatively large and problematic as to how they could be employed operationally.

For the moment, medical imaging is beyond the capabilities of most EDUs who have to make due with $\mathrm{x}$ ray equipment. However, the technology can be used to create the virtual IEDs for a game.

\subsection{Create representative IED}

To create a model from the DICOM data, we use 3D Slicer [26] to create and select label maps using threshold ranging. The ranges we chose were from -976.00 to 490.00. DICOM data captured from the MRI machine shows three hypothetical planes used to transect the entity; axial, sagittal and coronal.

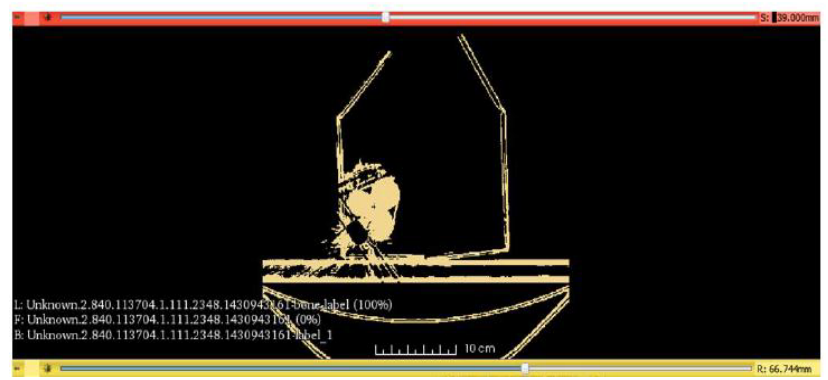

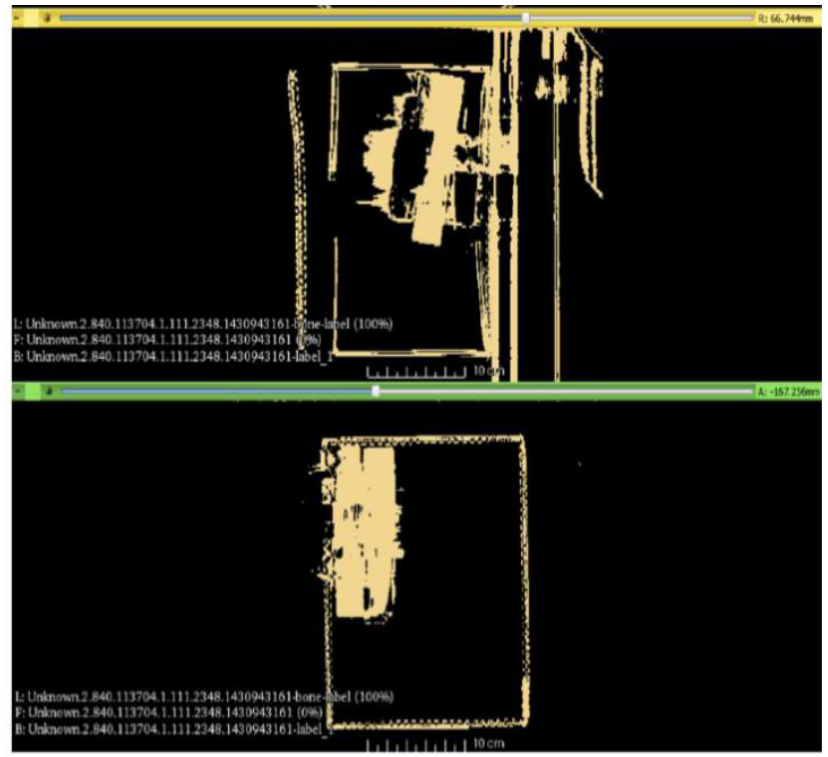

Figure 6. A representation of the cross sectional 3D Slicer.

The threshold feature in the editor allows for a user to choose what entities should be merged into a model.

However, the model created in 3D Slicer often includes artifacts which are caused by a variety of factors that may be related to scanning during motion, metallic components or foreign bodies [32]. Artifacts, as shown in figure 7 , can be identified as zippers, strips, fringes or overflow marks which can be caused by Finite sampling, k-space encoding, and Fourier transformation may cause aliasing and Gibbs artifacts [33].

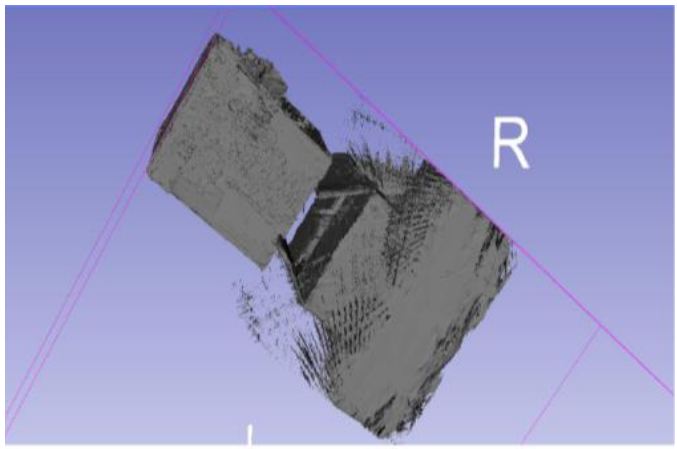

Figure 7. The 3D model produced by combining cross sections in $3 \mathrm{D}$ Slicer.

\subsection{Mesh cleanup}

To cleanup artifacts and extraneous entities such as the support surface, we use Blender [27] to select and delete parts of the mesh. We then use a hole filling algorithm [34] to generate a new surface mesh that follows the same shape as the input, but with more regular topology. The output of this algorithm is blocky re-meshes that are not too small or irregular--useful for separating into components in Aperio. The most important part of this process is to remove nonmanifolds-- geometries that cannot exist in the real world such as 3 or more planes intersecting on an edge. By using this algorithm, it also removes small disconnected pieces and fills holes in the mesh, the resulting mesh appears much like figure 8 . 


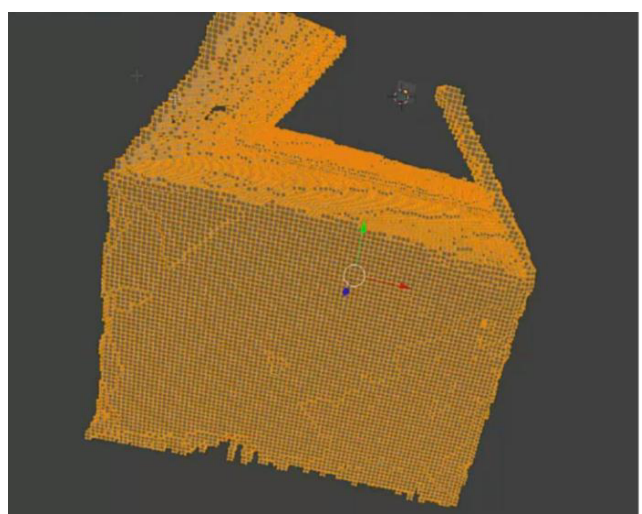

Figure 8. Mesh in Blender after using a hole filling algorithm.

\subsection{Mesh manipulation}

We identify key components by using Aperio [6], a mesh editor that manages 3D scene occlusion using tools similar to mechanical tools like a cutter, a ring and a rod to manipulate meshes. We identify which parts of the mesh visually represent IED components and separate them using these tools. The output is an object and material file compatible for importing into a game engine.

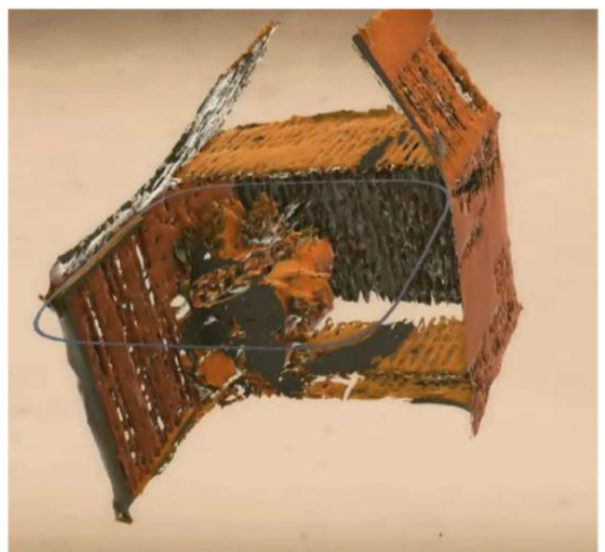

Figure 9. Mesh modified by tools in Aperio.

\subsection{Physics engine}

After importing models into the Unity game engine [28], we associate bounding boxes to certain components and assign unique scoring criteria to each box to indicate which are key regions of interest. The algorithm for the bounding box has three components-a damage receiver script, a bullet penetration script and a crosshair script.

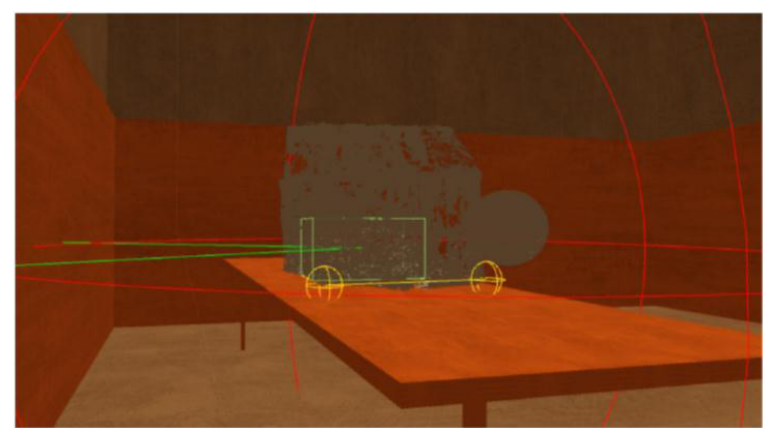

Figure 10. Visually associating a bounding box to the component object.

\subsection{Interaction with the game}

Inside the game, the player has a choice of picking up different models of disruptors. The objective of the game is to aim and shoot a projectile to neutralize the IED. As the projectile hits the object and its bounding box, an effectiveness score appears indicating whether it has successfully hit a key component or not. The player must look for visual cues shown within the bomb model. As the player hovers the crosshair near key components with the disruptor, the crosshair changes color, and as the projectile hits the bounding box, the distance and the angle to the target with respect to the normal plane of the disruptor are shown at the bottom of the display.

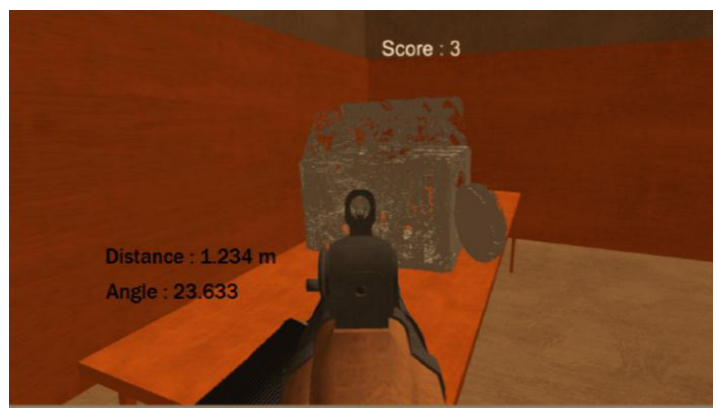

Figure 11. The 3D interactive game with the effectiveness score, distance and angle to the target with respect to the normal plane of the disruptor.

\section{Experiments and results}

We validated the simulation by comparing dimensions of the models in the simulation to dimensions of the model in the real-world. To determine the dimensions of the models in the game engine, we use a distance measuring tool [35] to measure game objects in the scene. This tool displays the ratio between object scale in pixels based on a user-defined pixel-per-unit setting.

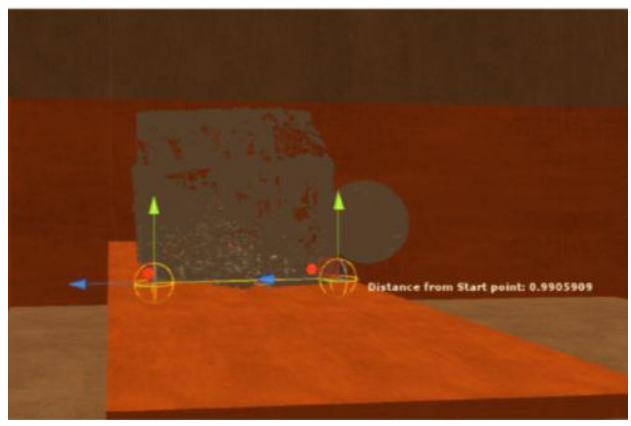

Figure 12. Distance tool from the Unity asset store to measure game objects in the scene.

The pixel-per-unit was set to $9.5714 \mathrm{px}=1 \mathrm{~cm}$, which means a pixel measurement of $100 \mathrm{px}$ would equal $10.4477 \mathrm{~cm}$. The results of our measurements are:

Table 1. Comparing Dimensions of the Container

\begin{tabular}{|c|c|c|}
\hline \multirow{2}{*}{$\begin{array}{l}\text { Sim vs. } \\
\text { Reality }\end{array}$} & \multicolumn{2}{|c|}{ Container Dimensions } \\
\cline { 2 - 3 } & Model in Sim & Model in Reality \\
\hline Height & $153.5224 \mathrm{px}=16.0397 \mathrm{~cm}$ & $16 \mathrm{~cm}$ \\
\hline Width & $201.6814 \mathrm{px}=21.0712 \mathrm{~cm}$ & $21 \mathrm{~cm}$ \\
\hline Length & $239.5571 \mathrm{px}=25.0284 \mathrm{~cm}$ & $25 \mathrm{~cm}$ \\
\hline
\end{tabular}


Table 2. Comparing Dimensions of the Bomb

\begin{tabular}{|c|c|c|}
\hline \multirow{2}{*}{$\begin{array}{c}\text { Sim vs. } \\
\text { Reality }\end{array}$} & \multicolumn{2}{|c|}{ Bomb Dimensions } \\
\cline { 2 - 3 } & Model in Sim & Model in Reality \\
\hline Height & $59.6987 \mathrm{px}=6.2372 \mathrm{~cm}$ & $6 \mathrm{~cm}$ \\
\hline Width & $67.1586 \mathrm{px}=7.0166 \mathrm{~cm}$ & $7 \mathrm{~cm}$ \\
\hline Length & $143.5744 \mathrm{px}=15.0003 \mathrm{~cm}$ & $15 \mathrm{~cm}$ \\
\hline
\end{tabular}

In Table 1, the comparison in terms of width suggest a $1.0033 \%$ difference when comparing the result of $21.0712 \mathrm{~cm}$ to $21 \mathrm{~cm}$. In Table II, the result for height shows a $1.0395 \%$ difference when comparing $6.2372 \mathrm{~cm}$ to $6 \mathrm{~cm}$. According to EDU professionals, they direct and aim disruptors (to the best of their ability) at the centre of their target. We suggest that we can provide a virtual model to accurately represent a real IED model within 1 $2 \%$ degree of difference.

\section{Conclusion and future work}

The motivation for our methodology has been to set the stage for safe investigation of IEDs for EDU professionals. In the field, EDU professionals use the Neutrex disruptor to fire high velocity water to separate the components of an IED before it explodes in an uncontrolled manner.

With our system, we simulate this by converting real world arbitrary models into virtual models and allow reasoning to occur about them, leading to game interaction and a score being produced - an inherently safe approach. We create and show an accurate representation of the real-world IED, and enable EDU professionals an additional framework that can provide them with information such as distances and angles from a safe virtual environment.

Our future work will include real-world validation by creating a situation where our game simulation is one of a real-world experimental rig involving an inert IED within a container and aiming and firing a real disruptor at one of the key components of the IED using only data available from our game simulation. In essence, we will use the game to inform our real-world decisions. If we hit the target, we win the game and our approach will be valid for at least one instance. If we miss the target, our score will be low or the game will end abruptly.

\section{References}

1. Department of Homeland Security (2015, Jun . 15). IED Attack Improvised Explosive Devices [Online]. Available:

https://www.dhs.gov/xlibrary/assets/prep_ied_fact_s heet.pdf.

2. A. Farazmand, Handbook of crisis and emergency management. CRC Press, 2001.

3. V. Smirnov, "Possibilities of Creating Improvised Explosives and Explosive Devices," in Detection and disposal of improvised explosives, Springer, 2006, pp. 217-221.

4. S. Mallonee, S. Shariat, G. Stennies, R. Waxweiler, D. Hogan, and F. Jordan, "Physical injuries and fatalities resulting from the Oklahoma City bombing," Jama, vol. 276, no. 5, pp. 382-387, 1996.

5. F. Craig (2015 Jun. 15) Anatomy of an IED. [Online]. Available

http://science.howstuffworks.com/ied1.htm

6. D. Tran (2015 May. 23) Aperio by eternallite, 3D Interactive Tool for Revealing Occlusions in Models [Online] Available http://eternallite.github.io/Aperio/

7. A. Ferworn, S. Herman, C. Kong, A. Ufkes, and J. Tran, "Interacting with a virtual Destroyed Environment constructed from real disaster data," in Safety, Security, and Rescue Robotics (SSRR), 2014 IEEE International Symposium on, 2014, pp. 1-6.

8. A. Ferworn, S. Herman, J. Tran, A. Ufkes, and R. Mcdonald, "Disaster scene reconstruction: Modeling and simulating urban building collapse rubble within a game engine," in Proceedings of the 2013 Summer Computer Simulation Conference, 2013, p. 18.

9. M. Coatsworth, J. Tran, and A. Ferworn, "A hybrid lossless and lossy compression scheme for streaming RGB-D data in real time," in Safety, Security, and Rescue Robotics (SSRR), 2014 IEEE International Symposium on, 2014, pp. 1-6.

10. Bureau of Alcohol, Tobacco, Firearms and Explosives (2015 Jun 3) ATF Explosives Handling Handbook [Online] Available https://www.atf.gov/file/58741/download

11. Arson. Enforcement (2015 Jun 15) Arson Explosives Enforcement [Online] Available https://www.atf.gov/content/Arson/arsonexplosivesenforcement

12. Scanna (2015 Jun 15) Scanna portable $x$-ray and postal $x$-ray systems. [Online] Available http://www.scanna-msc.com/uk/aboutscanna/

13. Proparms (2015 Jun 16) EOD Tools, Waterjet Disrupters and Forced Entry Tools - Proparms Ltd. [Online] Available http://www.proparms.com/site/product 10. html

14. R. Ratan and U. Ritterfeld, "Classifying serious games," Serious games: Mechanisms and effects, pp. 10-24, 2009.

15. C. C. Abt, Serious games. University Press of America, 1987.

16. M. Zyda, "From visual simulation to virtual reality to games," Computer, vol. 38, no. 9, pp. 25-32, 2005.

17. P. Rego, P. M. Moreira, and L. P. Reis, "Serious games for rehabilitation: A survey and a classification towards a taxonomy," in Information Systems and Technologies (CISTI), 2010 5th Iberian Conference on, 2010, pp. 1-6.

18. A. Seugling and M. Rölin, "Evaluation of physics engines and implementation of a physics module in a 3d-authoring tool," Umea University, 2, 2006.

19. A. Boeing and T. Bräunl, "Evaluation of real-time physics simulation systems," in Proceedings of the 5th international conference on Computer graphics and interactive techniques in Australia and Southeast Asia, 2007, pp. 281-288.

20. W. B. Stafford, "Sequential pattern detection and time series models for predicting IED attacks," Monterey, California. Naval Postgraduate School, 2009. 
21. D. E. Brown and M. Smith, "Terrorist Threat Prediction in Urban Environments."

22. L. Nilsen-Nygaard and C. W. Johnson, "Using mathematical models to guide the simulation of improvised explosive devices in public spaces," in System Safety, 2008 3rd IET International Conference on, 2008, pp. 1-6.

23. M. C. Association (2015 Jun 16) Counter-IED. [Online] Available https://www.mcamarines.org/gazette/2014/05/counter-ied

24. A. Shankar, "Spatial and Temporal Modeling of IED Emplacements against Dismounted Patrols," George Mason University, 2014.

25. S. Carpin, M. Lewis, J. Wang, S. Balakirsky, and C. Scrapper, "USARSim: a robot simulator for research and education," in Robotics and Automation, 2007 IEEE International Conference on, 2007, pp. 14001405 .

26. A. Fedorov, R. Beichel, J. Kalpathy-Cramer, J. Finet, J.-C. Fillion- Robin, S. Pujol, C. Bauer, D. Jennings, F. Fennessy, M. Sonka, and others, "3D Slicer as an image computing platform for the Quantitative Imaging Network," Magnetic resonance imaging, 30, no. 9, pp. 1323-1341, 2012.

27. B. O. Community, Blender - a $3 D$ modelling and rendering package. Blender Institute, Amsterdam: Blender Foundation.
28. Unity Technologies. (2014, Jul. 18). Unity Game Engine-Official Site [Online]. Available: http://unity3d. com

29. Epic Games. (2014, Jul. 18). Unreal Development Kit (UDK) [Online]. Available: https://www.unrealengine.com/products/udk/

30. D. Group, "Defense and Security Speciality Equipement.".

31. R. R. Edelman and S. Warach, "Magnetic resonance imaging," New England Journal of Medicine, 328, no. 10, pp. 708-716, 1993.

32. "MRI artifacts | Radiology Reference Article | Radiopaedia.org." .

33. E. M. Bellon, E. Haacke, P. Coleman, D. Sacco, D. Steiger, and R. Gangarosa, "MR artifacts: a review," American Journal of Roentgenology, 147, no. 6, pp. 1271-1281, 1986.

34. T. Ju, F. Losasso, S. Schaefer, and J. Warren, "Dual contouring of hermite data," ACM Transactions on Graphics (TOG), 21, no. 3, pp. 339-346, 2002.

35. W. McDermott (2015 Jun 28) Distance Tool. [Online] Available https://www.assetstore.unity3d.com/en/\#!/content/30 7 